\title{
Valerio Magrelli (a cura di), I linguaggi della guerra
}

\section{Francesca Forcolin e [francesca forcolin]}

\section{(C) OpenEdition}

\section{Journals}

\section{Edizione digitale}

URL: http://journals.openedition.org/studifrancesi/6490

DOI: 10.4000/studifrancesi.6490

ISSN: 2427-5856

\section{Editore}

Rosenberg \& Sellier

\section{Edizione cartacea}

Data di pubblicazione: 1 novembre 2010

Paginazione: 609-610

ISSN: 0039-2944

\section{Notizia bibliografica digitale}

Francesca Forcolin e [francesca forcolin], «Valerio Magrelli (a cura di), I linguaggi della guerra», Studi Francesi [Online], 162 (LIV | III) | 2010, online dal 30 novembre 2015, consultato il 11 janvier 2021. URL: http://journals.openedition.org/studifrancesi/6490 ; DOI: https://doi.org/10.4000/studifrancesi.6490

Questo documento è stato generato automaticamente il 11 janvier 2021.

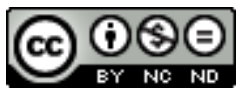

Studi Francesi è distribuita con Licenza Creative Commons Attribuzione - Non commerciale - Non opere derivate 4.0 Internazionale. 


\title{
Valerio Magrelli (a cura di), I linguaggi della guerra
}

\author{
Francesca Forcolin e [francesca forcolin]
}

\section{NOTIZIA}

VALERIO MAGRelLI (a cura di), I linguaggi della guerra, Santa Maria Capua Vetere, Edizioni Spartaco, 2009, pp. 228.

1 La ricerca sul tema della guerra e la sua rappresentazione letteraria in epoca moderna, oggetto del volume, nasce da una proposta formulata dal professor Nicola Bottiglieri, immediatamente accolta dal Dipartimento di Linguistica degli Studi di Cassino. L'idea traeva spunto da una giornata di studi tenutasi proprio a Cassino nel 2006, e vide via via la configurazione di un progetto che ha dato vita ai quattordici saggi pubblicati. Si tratta dell'indagine su modelli, forme e figure di rappresentazione della guerra nelle letterature di lingue e culture differenti, dalla Gran Bretagna alla Francia, dalla Russia all'Argentina, per un lasso di tempo che va dalla fine del Cinquecento al Novecento. Viene analizzata in tal modo l'evoluzione della concezione guerresca, da una visione classica, di tipo epico, a una visione dell'espressione dell'insensatezza esistenziale dell'umanità contemporanea.

2 Il saggio che apre l'opera, La guerra di Shakespeare di Maria VALENTINI (pp. 21-40), affronta il tema della guerra nelle opere del drammaturgo, proponendo un quadro complessivo dell'evoluzione delle sue posizioni, da un'iniziale giustificazione dei conflitti in epoca elisabettiana alla consapevolezza degli effettivi limiti.

3 Segue il contributo di Elisabetta SIBILIO, La madre di tutte le guerre: la guerra di Troia nel teatro di Jean Racine (pp. 41-58), dove si sottolinea l'evidente influsso di Omero nelle opere di Racine.

4 Sempre in ambito francese si svolge lo studio di Fiorella DI STEFANO, La guerra di Troia nel poema eroicomico da Tassoni a Perrault (pp. 59-68), dove si constata la metamorfosi del modello della guerra di Troia, da epico-classico a burlesco, tipico del periodo barocco. 
Dalla Querelle des Anciens et des Modernes prende le mosse Luciana PIRÉ, dedicando il suo intervento a Swift: Penne d'oca, inchiostro e vetriolo: "La battaglia dei libri" di Jonathan Swift (pp. 69-84).

5 Con Francesco PONTUALE e La battaglia di Lucy Terry prince (pp. 85-96) ci spostiamo nel Nuovo Mondo durante gli anni della guerra di successione austriaca (1744-48), con l'analisi della breve ballata Bars Fight, prima composizione in versi scritta in lingua inglese nel Nord America da un'autrice di origine africana.

6 Al maggiore rappresentante della cultura illuministica italiana è dedicato l'intervento di Angelo faBRIZI, Un illuminista tra gli "automi”. L'esperienza militare di Pietro Verri (pp. 97-112). Gabriele POOLE con La retorica della libertà nelle "Turkish Tales" (pp.113-126), indaga il concetto di militarismo in rapporto agli eroi byroniani, figure solitarie, cupe, condottieri-capi individualisti. A un autore contemporaneo di Byron è dedicato il contributo successivo, Chateaubriand, la gloria, il mattatoio: immagini di guerra nei "Mémoires d'outre-tombe" di Valerio MAGReLli (pp. 127-134), in cui si mette in luce la problematica della violenza e della guerra che occupano un posto saliente nei Mémoires, nonché l'atteggiamento del suo autore.

7 Ci spostiamo in Argentina nella seconda metà del XIX secolo con Amanda SALVIONI, che propone Con mano tremante d'ira: la guerra civile come problema di rappresentazione. Esteban Echeverría, "El matadero" (pp. 135-146); segue Il corpo smembrato di Nicola BOTTIGLIERI (pp. 147-162), analisi dei cimiteri di guerra nel periodo a cavallo tra le due Guerre Mondiali.

Nell'ambito della Seconda Guerra Mondiale si colloca l'interessante contributo di Annie OLIVIER, Écrire sur l'occupation en France (pp. 163-180): qui si constata come a partire dagli anni '70 diversi scrittori, attraverso autobiografie, autofictions, diari intimi e romanzi, abbiano riportato alla memoria scene della Guerra e dell'occupazione. Tra questi spiccano Patrick Modiano, in particolare col racconto Dora Bruder, e Marguerite Duras, oltre a Didier Daeninckx e Lydie Salvayre.

9 Ci spostiamo in Russia e alle campagne militari in Afghanistan e Cecenia con il contributo di Natalie MALININ, dal titolo Guerra, patria e "nuova" Russia nella letteratura russa della seconda metà del Novecento (pp. 181-198). Il penultimo articolo è di Ilaria MAGNANI, Le parole della guerra taciuta (pp. 199-216), che si sofferma sul recente conflitto tra inglesi e argentini nelle isole Malvine-Falkland.

Chiude il volume il contributo di Donatella ANTONELLIS, Scrivere la guerra giorno per giorno (pp. 217-228), analisi di alcuni diari francesi degli ultimi dieci anni (tra gli altri, La vie extérieure di Annie Ernaux e L'autre faim di Charles Juliet) che trattano il conflitto bellico, da quelli a maggior risonanza internazionale a quelli di minore intensità.

11 Gli articoli sono accuratamente presentati dalla Premessa di Amanda SALVIONI (pp. 5-8) e dall'Introduzione di Valerio MAGRELLI (pp. 9-20). 